An elementary proof of Wallis' product formula for pi

\author{
JOHAN WÄSTLUND
}

Linköping studies in Mathematics, No. 2, February 21, 2005

Series editor: Bengt Ove Turesson 
The publishers will keep this document on-line on the Internet (or its possible replacement network in the future) for a period of 25 years from the date of publication barring exceptional circumstances as described separately.

The on-line availability of the document implies a permanent permission for anyone to read, to print out single copies and to use it unchanged for any noncommercial research and educational purpose. Subsequent transfers of copyright cannot revoke this permission. All other uses of the document are conditional on the consent of the copyright owner. The publication also includes production of a number of copies on paper archived in Swedish University libraries and by the copyright holder(s). The publisher has taken technical and administrative measures to assure that the on-line version will be permanently accessible and unchanged at least until the expiration of the publication period.

For additional information about the Linköping University Electronic Press and its procedures for publication and for assurance of document integrity, please refer to its WWW home page: http://www.ep.liu.se.

Linköping studies in mathematics, No. 2 (2005)

Series editor: Bengt Ove Turesson

Department of Mathematics (MAI)

http://math.liu.se/index-e.html

Linköping University Electronic Press

Linköping, Sweden, 2005

ISSN 0348-2960 (print)

www.ep.liu.se/ea/lsm/2005/002/ (www)

ISSN 1652-4454 (on line)

(c) Johan Wästlund. 


\title{
AN ELEMENTARY PROOF OF WALLIS' PRODUCT FORMULA FOR PI
}

\author{
JOHAN WÄSTLUND
}

\begin{abstract}
We give an elementary proof of the Wallis product formula for pi. The proof does not require any integration or trigonometric functions.
\end{abstract}

\section{The Wallis Product formula}

In 1655 , John Wallis wrote down the celebrated formula

$$
\frac{2}{1} \cdot \frac{2}{3} \cdot \frac{4}{3} \cdot \frac{4}{5} \cdots=\frac{\pi}{2} .
$$

Most textbook proofs of (1) rely on evaluation of some definite integral like

$$
\int_{0}^{\pi / 2}(\sin x)^{n} d x
$$

by repeated partial integration. The topic is usually reserved for more advanced calculus courses. The purpose of this note is to show that (1) can be derived using only the mathematics taught in elementary school, that is, basic algebra, the Pythagorean theorem, and the formula $\pi \cdot r^{2}$ for the area of a circle of radius $r$.

Viggo Brun gives an account of Wallis' method in [1] (in Norwegian). Yaglom and Yaglom [2] give a beautiful proof of (1) which avoids integration but uses some quite sophisticated trigonometric identities.

\section{A NUMBER SEQUENCE}

We denote the Wallis product by

$$
W=\frac{2}{1} \cdot \frac{2}{3} \cdot \frac{4}{3} \cdot \frac{4}{5} \cdots .
$$

The partial products involving an even number of factors form an increasing sequence, while those involving an odd number of factors form a decreasing sequence. We let $s_{0}=0, s_{1}=1$, and in general,

$$
s_{n}=\frac{3}{2} \cdot \frac{5}{4} \cdots \frac{2 n-1}{2 n-2} .
$$

The partial products of (2) with an odd number of factors can be written as

$$
\frac{2 n}{s_{n}^{2}}=\frac{2^{2} \cdot 4^{2} \cdots(2 n)}{1 \cdot 3^{2} \cdots(2 n-1)^{2}}>W,
$$

while the partial products with an even number of factors are of the form

$$
\frac{2 n-1}{s_{n}^{2}}=\frac{2^{2} \cdot 4^{2} \cdots(2 n-2)^{2}}{1 \cdot 3^{2} \cdots(2 n-3)^{2} \cdot(2 n-1)}<W .
$$

It follows that

$$
\frac{2 n-1}{W}<s_{n}^{2}<\frac{2 n}{W} .
$$

Date: February 21, 2005. 
We denote the difference $s_{n+1}-s_{n}$ by $a_{n}$, and observe that

$$
a_{n}=s_{n+1}-s_{n}=s_{n}\left(\frac{2 n+1}{2 n}-1\right)=\frac{s_{n}}{2 n}=\frac{1}{2} \cdot \frac{3}{4} \cdots \frac{2 n-1}{2 n} .
$$

We first derive the identity

$$
a_{i} a_{j}=\frac{j+1}{i+j+1} a_{i} a_{j+1}+\frac{i+1}{i+j+1} a_{i+1} a_{j} .
$$

Proof. After the substitutions

$$
a_{i+1}=\frac{2 i+1}{2(i+1)} a_{i}
$$

and

$$
a_{j+1}=\frac{2 j+1}{2(j+1)} a_{j}
$$

the right hand side of (4) becomes

$$
a_{i} a_{j}\left(\frac{2 j+1}{2(j+1)} \cdot \frac{j+1}{i+j+1}+\frac{2 i+1}{2(i+1)} \cdot \frac{i+1}{i+j+1}\right)=a_{i} a_{j} .
$$

If we start from $a_{0}^{2}$ and repeatedly apply (4), we obtain the identities

(5) $1=a_{0}^{2}=a_{0} a_{1}+a_{1} a_{0}=a_{0} a_{2}+a_{1}^{2}+a_{2} a_{0}=\ldots$

$$
\cdots=a_{0} a_{n}+a_{1} a_{n-1}+\cdots+a_{n} a_{0} .
$$

Proof. By applying (4) to every term, the sum $a_{0} a_{n-1}+\cdots+a_{n-1} a_{0}$ becomes

$$
\left(a_{0} a_{n}+\frac{1}{n} a_{1} a_{n-1}\right)+\left(\frac{n-1}{n} a_{1} a_{n-1}+\frac{2}{n} a_{2} a_{n-2}\right)+\cdots+\left(\frac{1}{n} a_{n-1} a_{1}+a_{n} a_{0}\right) .
$$

After collecting terms, this simplifies to $a_{0} a_{n}+\cdots+a_{n} a_{0}$.

\section{A geometric CONSTRUCtion}

We divide the positive quarter of the $x$-y-plane into rectangles by drawing the straight lines $x=s_{n}$ and $y=s_{n}$ for all $n$. Let $R_{i, j}$ be the rectangle with lower left corner $\left(s_{i}, s_{j}\right)$ and upper right corner $\left(s_{i+1}, s_{j+1}\right)$. The area of $R_{i, j}$ is $a_{i} a_{j}$. Therefore the identity (5) states that the total area of the rectangles $R_{i, j}$ for which $i+j=n$ is 1 . We let $P_{n}$ be the polygonal region consisting of all rectangles $R_{i, j}$ for which $i+j<n$. Hence the area of $P_{n}$ is $n$ (see Figure 1).

The outer corners of $P_{n}$ are the points $\left(s_{i}, s_{j}\right)$ for which $i+j=n+1$. By the Pythagorean theorem, the distance of such a point to the origin is

$$
\sqrt{s_{i}^{2}+s_{j}^{2}} \text {. }
$$

By (3), this is bounded from above by

$$
\sqrt{\frac{2(i+j)}{W}}=\sqrt{\frac{2(n+1)}{W}} .
$$

Similarly, the inner corners of $P_{n}$ are the points $\left(s_{i}+s_{j}\right)$ for which $i+j=n$. The distance of such a point to the origin is bounded from below by

$$
\sqrt{\frac{2(i+j-1)}{W}}=\sqrt{\frac{2(n-1)}{W}} .
$$




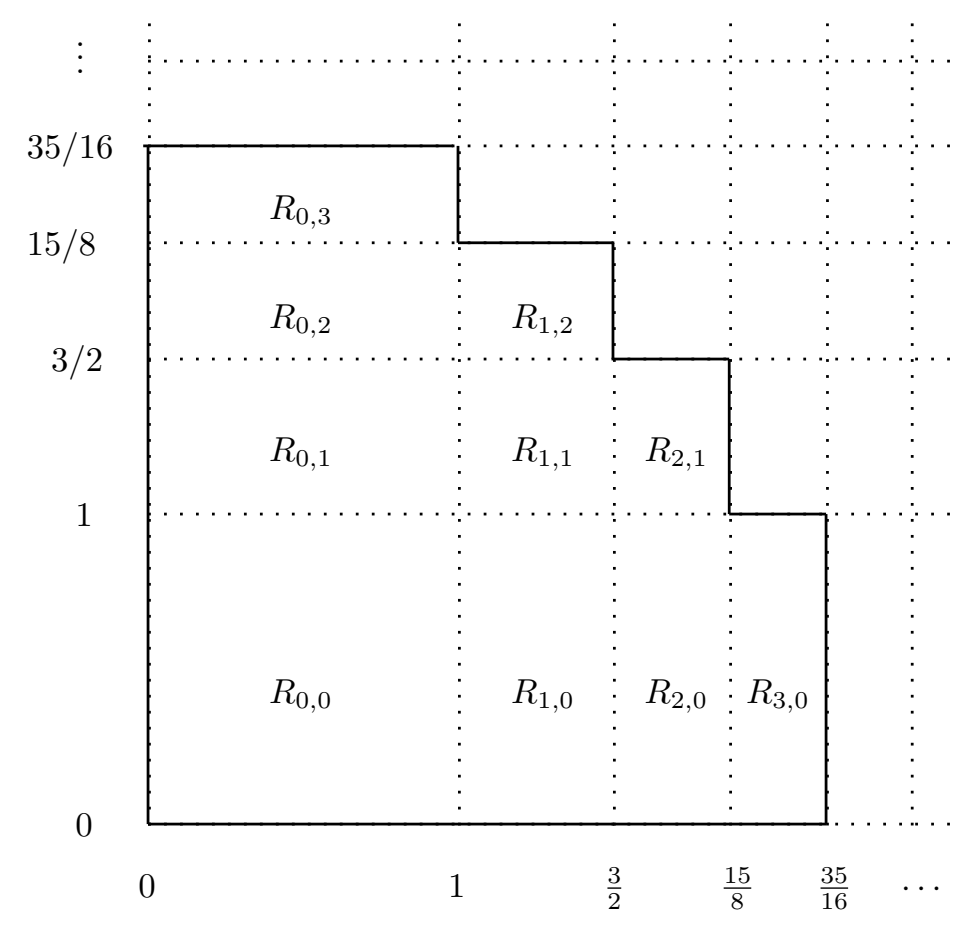

Figure 1. The region $P_{4}$ of area 4.

Therefore $P_{n}$ contains a quarter circle of radius $\sqrt{2(n-1) / W}$, and is contained in a quarter circle of radius $\sqrt{2(n+1) / W}$. Since the area of a quarter circle of radius $r$ is equal to $\pi r^{2} / 4$, we obtain the following bounds for the area of $P_{n}$ :

$$
\frac{\pi(n-1)}{2 W}<n<\frac{\pi(n+1)}{2 W} .
$$

Since this holds for every $n$, we conclude that

$$
W=\frac{\pi}{2} .
$$

\section{REFERENCES}

[1] Brun, Viggo., Wallis's og Brounckers formler for $\pi$ (in Norwegian), Norsk matematisk tidskrift, 33 (1951) 73-81.

[2] Yaglom, A. M. and Yaglom, I. M., An elementary derivation of the formulas of Wallis, Leibnitz and Euler for the number $\pi$ (in Russian), Uspechi matematiceskich nauk. (N. S.) 8 , no. 5 (57) (1953), 181-187. 